N. E. Piskunov, W. W. Weiss, D. F. Gray, eds.

\title{
Interferometric Observations of Mira Variables
}

\author{
G. van Belle, R.R. Thompson, M.J. Creech-Eakman \\ Jet Propulsion Laboratory, California Institute of Technology, \\ Pasadena, CA 91109
}

\begin{abstract}
Milliarcsecond resolution observations of cool stars are becoming increasingly common and sophisticated as recent advances in telescope technology mature. To varying degrees, these observations rely up on stellar models for interpretation of their data, while at the same time present particular challenges to those models. Indications of departures from spherical symmetry are beginning to be observed as increasingly rich image information is obtained by a new generation of interferometers. Examination the subtle variations of wavelength-specific sizes exhibits rich structure, connected to the atmospheric chemistry. For the pulsating stars, such as Mira variables, that structure varies with time, with the phase lags between the various sizes being connected to the atmospheric dynamics. Complex morphologies associated with atmospheric winds have been revealed with these high resolution experiments. A review of these recent results will be presented, concentrated on their implications upon stellar modelling, and the prospects for future observational data.
\end{abstract}

\section{Introduction}

High resolution observations of Mira variables have been performed for over 25 years, exploiting a variety of interferometric techniques. In 1973 Bonneau and Laberyie observed Mira itself using the new field of speckle interferometry, and followed up that measurement with additional ones of $\mathrm{R}$ Leo (Laberyie et al 1977). Mira was also observed early on using aperture masking techniques (McCarthy, Howell \& Low 1978) and was one of the first targets of the early generation of modern long-baseline interferometers (Ridgway et al 1992). Additionally, lunar occultations have been utilized for measuring diameters of Miras, beginning with U Ori in 1977 (Ridgway, Wells \& Joyce 1977). In recent years, a substantial body of Mira diameter measurements has been published using the Infrared-Optical Telescope Array (IOTA; van Belle et al 1996, 1997, Perrin et al 1999, Hofmann et al 2002, van Belle, Thompson \& Creech-Eakman 2002).

\section{Observations}

The predominant high-resolution methods in present use on Miras currently are lunar occultations, aperture masking \& speckle interferometry, and long-baseline interferometry. Each of these three techniques benefits from advances over the 


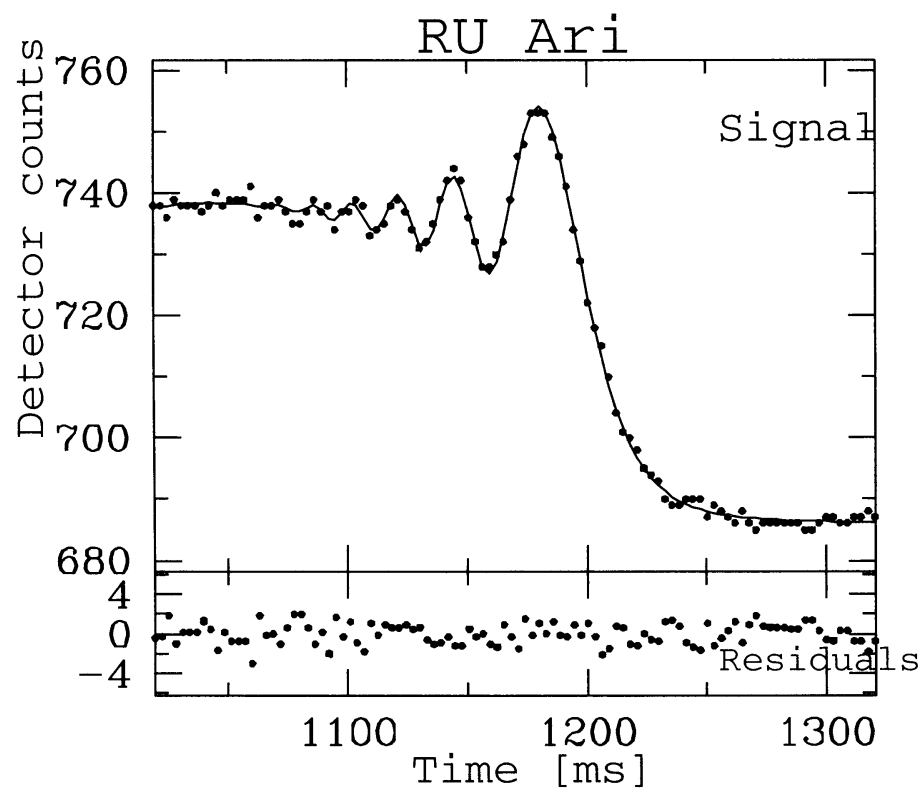

Figure 1. Lunar occultation of RU Ari from Richichi et al 1998.

last 25 years in detector technology, computing power, and optomechanical design.

\subsection{Lunar Occultations}

First among the techniques to achieve milliarcsecond resolution levels, practitioners of the lunar occultation technique have enjoyed a two decade head start on the technique of long baseline interferometery, the only other approach that yields information at that spatial scale. The lunar occultation technique utilizes the lunar disk as an occulting mask, with high-frequency sampling (on the order of a millisecond per sample) of the stellar brightness measuring the diffraction pattern as the star disappears behind the lunar disk. Conversely, re-emergence of the object from behind the lunar disk can also be used. The measured diffraction pattern is then compared to a model that yields the size of the star. An example of an occultation measure is illustrated in Figure 1.

Considerable amounts of early work can be found in the literature from Ridgway and his co-workers (eg. Ridgway et al 1977), and the leading modern effort is associated with Richichi and his co-workers (eg. Richichi et al 1998, Tej et al 1999, Richichi \& Calamai 2001).

Key limitations associated with lunar occultations are limited sky coverage, limited epoch coverage, and only a single axis of spatial information results from the measurement. However, within those limitations, information from lunar occultations has provided parameterizations of key stellar features that were, until only recently, inaccessible by any other means. 


\subsection{Aperture Masking \& Speckle Interferometry}

Aperture masking is a technique by which a single large aperture is transformed into an $N$-element interferometer by means of a pupil mask. Although the resultant interferometer's spatial resolution is limited to the physical extent of the base aperture, the number subaperture elements $N$ can be quite large, resulting in a relative wealth of interferometric information on the target being observed, in the form of closure phases and amplitudes. Most recently, the Keck 1 telescope has been utilized in this fashion to observe targets such as Mira, $\alpha$ Ori, $\alpha$ Tau, and W Hya. A thorough review of Keck aperture masking, including J, H, $\mathrm{K}$ and L band observations of Mira, is given Tuthill, Monnier \& Danchi (1999), and recent results on CIT 6 and IRC+10216 are decidedly impressive (Monnier et al 2000, Tuthill et al 2000). This technique is somewhat limited in that the observed targets need to be relatively bright and large; for the Keck, the resolution limit is on the order of 20 milliarcseconds. The brightness limit is dictated by the atmosphere, not the aperture size: each subaperture in the pupil mask must match the atmospheric isoplanatic patch size, and each frame integration time must be no longer than an atmospheric coherence time. On Mauna Kea, these values are typically on order 20 centimeters and 20 milliseconds, respectively, limiting targets to brightnesses greater than $K<4$.

Similarly, speckle interferometry on large apertures has been utilized to garner high-resolution information on some of the large prototypes of evolved stars. Notable in this field in recent years is the work published by Weigelt and his co-workers using the Special Astrophysical Observatory 6-m telescope (eg. Weigelt et al 2002, Yudin et al 2001).

\subsection{Long Baseline Interferometry}

The predominant current technique for examining Mira variables at high resolution is long-baseline interferometry (LBI). Currently there are three groups carrying out active research programs ('active' being loosely defined as having a refereed Mira publication in the last 4 years): the Cambridge Optical Aperture Synthesis Telescope group (COAST; Young et al 2000), users of the IR/Optical Telescope Array telescope (IOTA; van Belle, Thompson, \& CreechEakman 2002) and the associated Fiber Linked Unit for Optical Recombination instrument group (FLUOR; Hofmann et al 2002), and the Palomar Testbed Interferometer Collaboration (PTI; Thompson, Creech-Eakman \& van Belle 2002). Aside from producing a mouthful of acronyms, between $50 \%$ and $75 \%$ of the high-resolution observations of Miras in the published literature have been conducted with LBI (depending slightly upon how the tally is performed, Richichi \& Percheron 2002).

\section{Observations of Miras with the Palomar Testbed Interferometer}

The Palomar Testbed Interferometer (PTI) is a three-element near-infrared interferometer located atop Palomar Mountain next to the historic 5-meter Hale telescope. The 40-cm siderostat apertures are combined in a pairwise fashion to obtain, track, and record stellar fringes on 110-m and 85-m baselines, in the $\mathrm{H}$ - and K-bands. Details regarding the interferometer and its operation can 
be found in Colavita et al (1999) and Colavita (1999); relevant early results with the instrument include giant and supergiant diameters found in van Belle et al (1999). PTI is capable of measuring objects with angular sizes between 1 mas $<\theta<5$ mas, brighter than $\mathrm{K}<5$ or $\mathrm{H}<3.5$ in the near-infrared (for fringe tracking), and $\mathrm{R}<9$ in the visible (for tip-tilt tracking). PTI was built as a technology testbed for the Keck Interferometer, but has also served admirably as a scientific instrument in its own right.

Since 1998, a long term program of observing Mira diameters has been carried out, resulting in the accumulation of rich set of data on these objects. Over the past 5 years, diameters for over 50 Miras have been measured, with more than 30 of them being observed 10 or more times.

The PTI observations have typically been taken in the K band, which PTI records as both a broadband measurement across the entire band, and as five "narrowband" measures centered at 2.009, 2.106, 2.203, 2.299, and $2.396 \mu \mathrm{m}$. These narrowband diameters are of particular interest in attempting to decipher the angular size information from the interferometer, in that they sample decidedly different regions of the atmosphere. On both edges of the K-band, $\mathrm{H}_{2} \mathrm{O}$ can be seen to impact the spectra for the oxygen rich Miras. For the long wavelength edge of $\mathrm{K}$-band, $\mathrm{CO}$ manifests itself in a dominant fashion, particularly in the carbon-rich stars, along with $\mathrm{HCN}$ and $\mathrm{C}_{2} \mathrm{H}_{2}$. In conjunction with the high spatial resolution, increasing the spectral resolution has the potential to greatly expand the utility of interferometric observations in unravelling the atmospheric structure and dynamics of these objects.

Illustrating the particulars of the data is Figure 2, which overlaps angular size data for carbon-rich and oxygen-rich Miras and non-Miras with corresponding spectra data from Lançon \& Wood (2000). Furthermore, with the dynamic nature of Mira variables, the relationship of the individual narrowband sizes with respect to each other varies over the course of the star's cycle. The nature of this relationship is dependant upon the chemical abundance of the star, as seen in Figure 3 for the oxygen-rich Mira S Lac, and the carbon-rich Mira RZ Peg. The details of the interesting similarities and differences of these plots (particularly the phase lags) are discussed in detail in Thompson, Creech-Eakman \& van Belle (2002).

These plots are particularly interesting for examining the periodic oscillations of the stars since they are multi-periodic observations of individual objects. Since the distances to Miras continue to suffer from poor calibration (as discussed in van Belle, Thompson, \& Creech-Eakman 2002), meaningful comparisons of sizes as a function of phase is only possible for individual objects, relative to themselves. As is illustrated in Figure 4 with yet-to-be published PTI data on the Mira R Boo, multiple epochs of observations can serve to characterize the evolution of basic parameters such as radius and temperature.

\section{Stellar Modelling and High Resolution Observations}

\subsection{What Can High Resolution Observations Do For Modelling?}

High resolution imaging has the potential to provide size and surface structure information as a function of phase and wavelength. Gross sizes in multiple axes across the stellar disk can be obtained at an accuracy level of $1-10 \%$. Current 
Thompson, Creech-Eakman, van Belle (2002)

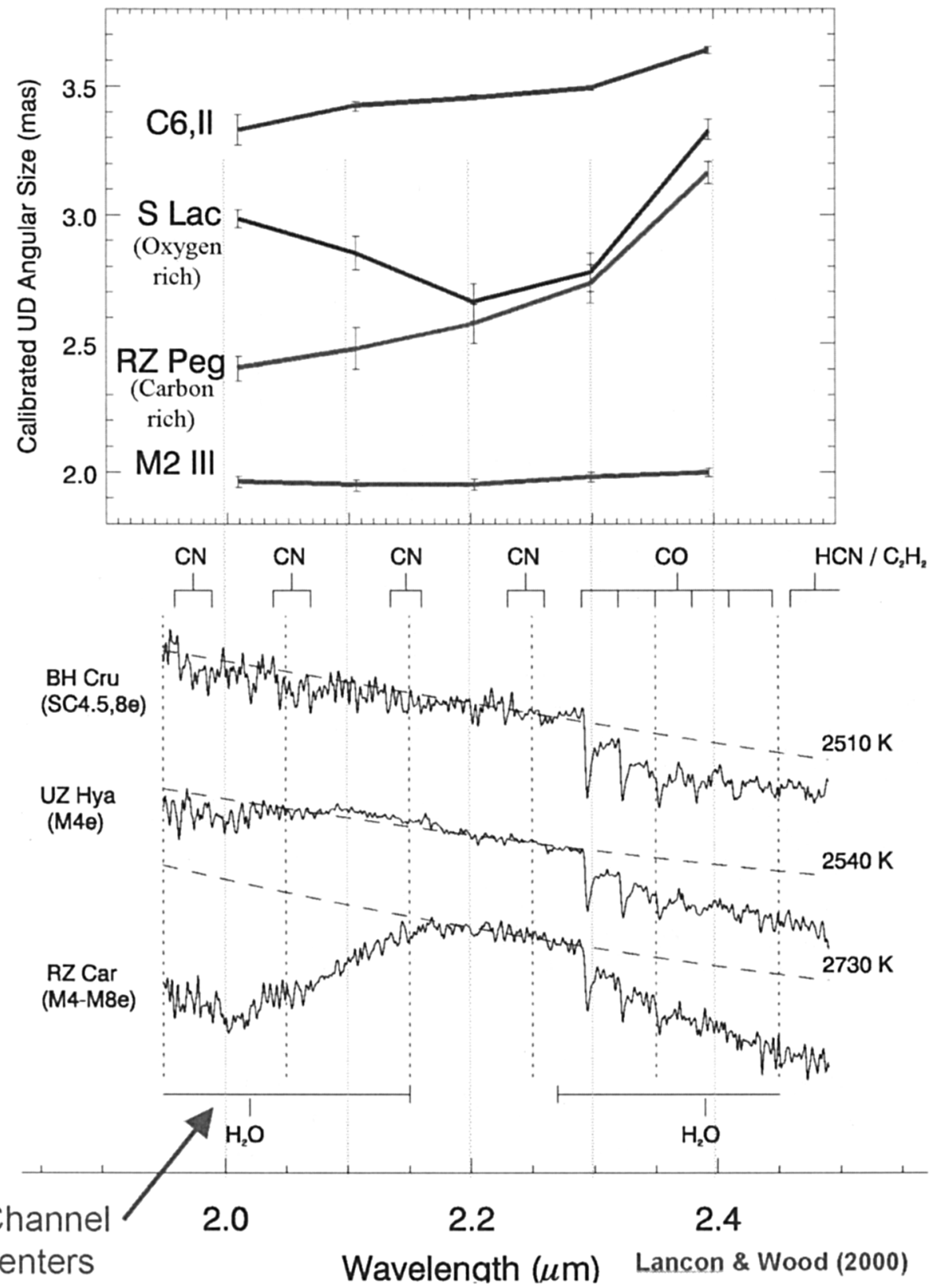

Figure 2. Comparison of the PTI narrowband sizes from Thompson, Creech-Eakman \& van Belle (2002) to the spectral information found in Lançon \& Wood (2000), for oxygen-rich and carbon-rich Miras and non-Mira stars. 

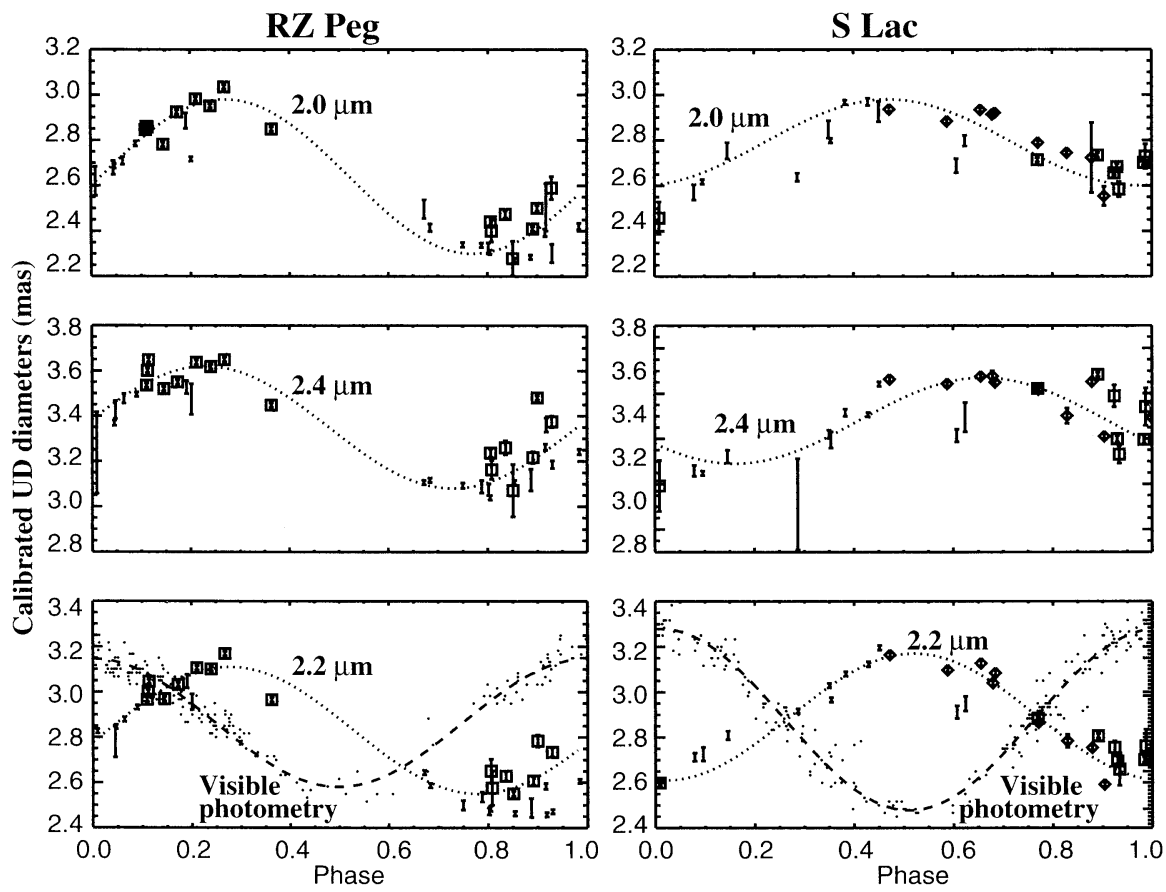

Figure 3. Narrowband size variations of the oxygen-rich Mira S Lac and the carbon-rich Mira RZ Peg as discussed in Thompson, CreechEakman \& van Belle (2002). The bottom panel for each star also displays the contemporaneous visible light photometry. 


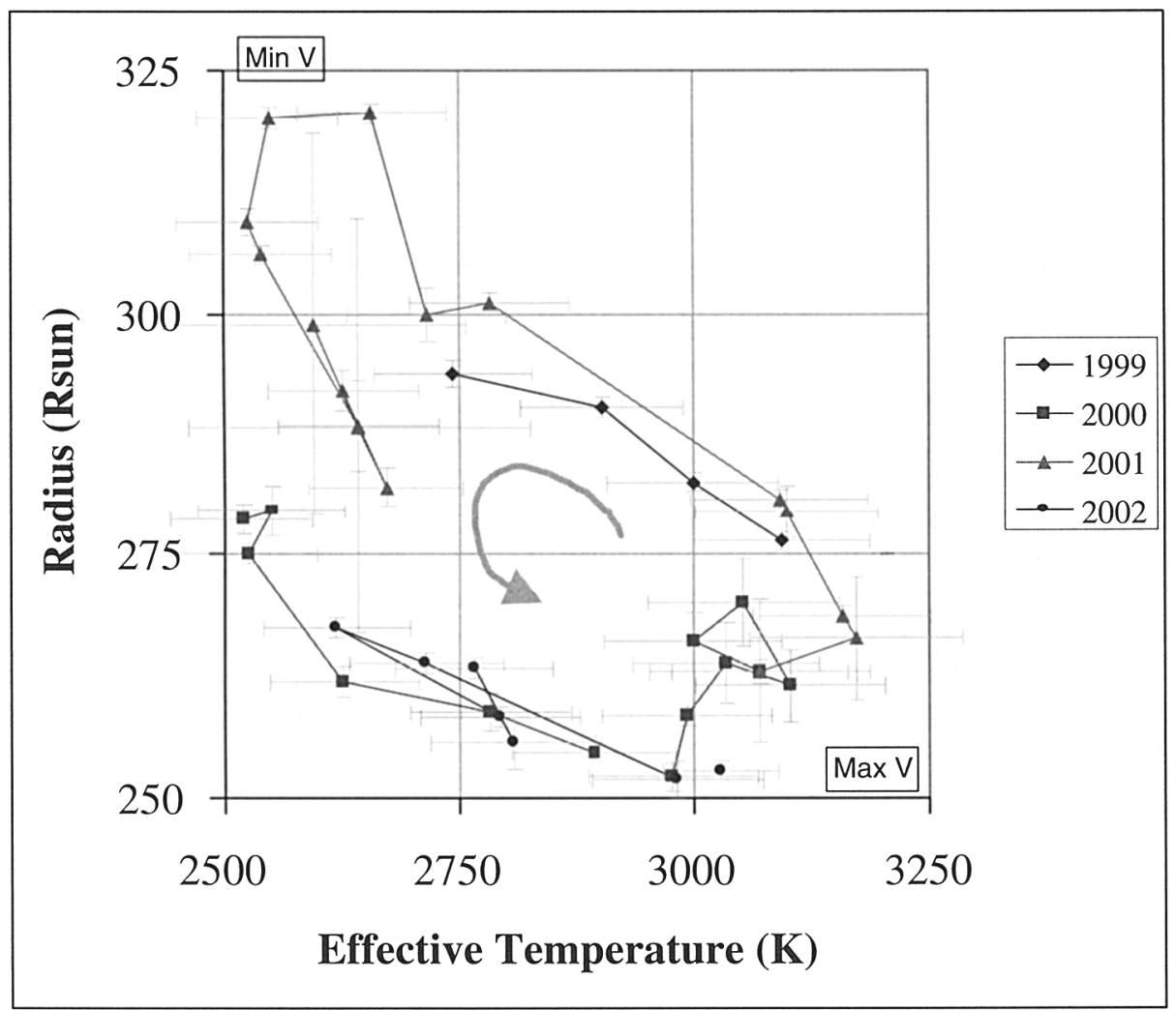

Figure 4. Stellar radius versus temperature for the Mira R Boo, from presently unpublished PTI data. The lower right of the plot corresponds to stellar maximum light, while the upper left corresponds to minimum light. Over the course of its cycle, the star traces out a counter-clockwise path upon this radius-temperature plot. Within the precision of the indicated errors, the star retraces its path on this plot repeatedly from cycle to cycle. 
spectral resolutions are $R \approx 20$, and higher resolution $R \approx 200$ systems are ungoing testing. While these spectral resolutions fall far short of the $R>10,000$ systems on single aperture telescopes, one must not forget that these spectra are coupled with spatial information, making them very powerful tools for probing stellar structure. Existing systems have been monitoring a large $(>40)$ sample of Miras throughout their cycles, and significantly larger aperture interferometers are imminent (see Table 1).

Further down the road (in 5-10 years), interferometric astrometry has the potential to provide direct measurements of Mira distances. These distances will have $1-5 \%$ accuracies out to about a kiloparsec, which should be sufficient to drive the final nails in the coffin of the pulsation mode question for Mira variables.

\subsection{What Can Modelling Do For High Resolution Observations?}

In many cases, the capabilities of particular high resolution techniques exceeds the ability or resources of its most intimate users to most adequately exploit them. Practicioners of the black arts of stellar modelling would do well to identify the "sweet spots" where particular high resolution measurements can help constrain or guide models. Towards that end, it is incumbent upon the interferometrists to teach modelers about the capabilities and limitations of their instruments, and hopefully this manuscript is a step in that direction. In particular, Table 1 attempts to identify the relevant parameters to be aware of when designing observing programs. Once so informed, modelers should identify realistic tests of greatest utility to help validate their models. Two recent papers by Scholz (Scholz 2001, Jacob \& Scholz 2002) illustrate a useful approach to models that takes the final constructions and presents them in a fashion that make then directly applicable to interferometric studies.

Acknowledgments. We are grateful to Lee Anne Willson for graciously extending an invitation to come to the Stellar Modelling symposium. We would also like to thank the gracious Palomar Mountain staff and PTI Collaboration for their support of this observing program. Funding for PTI was provided to the Jet Propulsion Laboratory under its TOPS (Towards Other Planetary Systems), ASEPS (Astronomical Studies of Extrasolar Planetary Systems), and Origins programs and from the JPL Director's Discretionary Fund. Portions of this work were performed at the Jet Propulsion Laboratory, California Institute of Technology under contract with the National Aeronautics and Space Administration.

\section{References}

Bonneau, D., \& Labeyrie, A., 1973, ApJ, 181, 1

Colavita, M.M., 1999, PASP, 111, 111

Colavita, M.M., et al, 1999, ApJ, 510, 505

Hofmann, K.-H., et al, 2002, New Astronomy, 7, 9

Jacob, A.P., \& Scholz, M., 2002, MNRAS, in press 
Table 1. Capabilities and limitations of current and upcoming highresolution facilities.

\begin{tabular}{|c|c|c|c|c|c|c|}
\hline Technique & Instrument & $\begin{array}{c}\text { Brightness } \\
\text { limit }\end{array}$ & $\begin{array}{l}\text { Other } \\
\text { limits }^{g}\end{array}$ & $\begin{array}{l}\text { Target Sizes } \\
\text { (mas) }\end{array}$ & Bands & Elements \\
\hline $\begin{array}{l}\text { Lunar } \\
\text { Occultation }^{a}\end{array}$ & TIRGO & $\mathrm{K}<3$ & & $>1.4$ & JHK & $\begin{array}{c}\text { Effectively } \\
2 \\
\end{array}$ \\
\hline $\begin{array}{l}\text { Aperture } \\
\text { Masking }\end{array}$ & Keck 1 & $\mathrm{~K}<4$ & & $>20$ & JHKL & $>20^{b}$ \\
\hline $\begin{array}{l}\text { Speckle } \\
\text { Interferometry }\end{array}$ & SAO $6 \mathrm{~m}$ & $\mathrm{~K}<4 ?$ & & $>33$ & VRJHK & $\begin{array}{l}\text { Effectively } \\
\text { many }^{b}\end{array}$ \\
\hline Long Baseline & PTI & $\mathrm{K}<5$ & $\mathrm{R}<9$ & $1.5-5.0$ & $\mathrm{HK}^{c}$ & 2 \\
\hline Interferometry & $\begin{array}{l}\text { IOTA } \\
\text { COAST }\end{array}$ & $\begin{array}{l}\mathrm{K}<6 \\
\mathrm{~K}<5 ?\end{array}$ & $\begin{array}{l}\mathrm{R}<9 ? \\
\mathrm{R}<9 ?\end{array}$ & $\begin{array}{l}4.5-30 \\
3.5-70\end{array}$ & $\begin{array}{l}\text { JHKL } \\
\text { RIJHK }\end{array}$ & $\begin{array}{l}3 \\
5\end{array}$ \\
\hline $\begin{array}{l}\text { Upcoming } \\
\text { facilities }\end{array}$ & $\begin{array}{l}\text { Keck outriggers }^{d, f} \\
\text { VLTI auxiliary } \\
\text { telescopes }^{e, f}\end{array}$ & $\begin{array}{l}K<10 \\
K<10\end{array}$ & $\begin{array}{c}\mathrm{J}<10 \\
\text { unknown }\end{array}$ & $\begin{array}{c}1.2-6 \\
0.9-30\end{array}$ & $\begin{array}{l}\text { JHK } \\
\text { JHK }\end{array}$ & $\begin{array}{l}4 \\
3\end{array}$ \\
\hline
\end{tabular}

${ }^{a}$ Requires chance conjunction of target, moon, and covers a limited range of declinations.

${ }^{b}$ Rich image reconstructions.

${ }^{c} \mathrm{R}=20(\mathrm{R}=100-200$ being tested $)$.

${ }^{d}$ Online in 2004.

${ }^{e}$ Online in 2003.

${ }^{f}$ To be open for general observing proposals.

${ }^{g}$ Typically dictated by the instrument's tip-tilt sensor.

Labeyrie, A., Koechlin, L., Bonneau, D., Blazit, A., \& Foy, R., 1977, ApJ, 218, 75

Lançon, A., \& Wood, P.R., 2000, A\&AS, 146, 217

McCarthy, D.W., Howell, R.R., \& Low, F.J., 1978, ApJ, 223, 113

Monnier, J.D., Tuthill, P.G., \& Danchi, W.C., 2000, ApJ545, 957

Perrin, G., Coudé du Foresto, V., Ridgway, S.T., Mennesson, B., Ruilier, C., Mariotti, J.-M., Traub, W.A., \& Lacasse, M.G., 1999, A\&A, 345, 221

Richichi, A., \& Calamai, G., 2001, A\&A, 380, 562

Richichi, A., \& Percheron, I., 2002, A\&A, 386, 492

Richichi, A, Ragland, S., Stecklum, B., \& Leinert, C., 1998, A\&A338 527

Ridgway, S.T., Benson, J.A., Dyck, H.M., Townsley, L.K., \& Hermann, R.A., 1992, AJ, 104, 2224

Ridgway, S.T., Wells, D.C., \& Joyce, R.R., 1977, AJ, 82, 414

Tej, A., Chandrasekhar, T., Ashok, N.M., Ragland, S., Richichi, A., \& Stecklum, B., 1999, AJ, 117, 1857

Scholz, M., 2001, MNRAS, 321, 347

Thompson, R.R., Creech-Eakman, M.J., \& van Belle, G.T., 2002, ApJ, 577, 447

Tuthill, P.G., Monnier, J.D., Danchi, W.C., 1999, Working on the Fringe: Optical and IR Interferometry from Ground and Space, Proceedings from ASP Conference Vol. 194, Stephen Unwin \& Robert Stachnik eds., 188 
Tuthill, P.G., Monnier, J.D., Danchi, W.C., \& Lopez, B., 2000, ApJ543, 284 van Belle, G.T., Dyck, H.M., Benson, J.A., \& Lacasse, M.G., 1996, AJ, 112, 2147

van Belle, G.T., Dyck, H.M., Thompson, R.R., Benson, J.A., \& Kannappan, S.J., 1997, AJ, 114, 2150

van Belle, G.T., et al, 1999, AJ, 117, 521

van Belle, G.T., Thompson, R.R., \& Creech-Eakman, M.J., 2002, AJ, 124, 1706

Weigelt, G., Balega, Y.Y., Blöcker, T., Hofmann, K.-H., Men'shchikov, A.B., \& Winters, J.M., 2002, A\&A392 131

Young, J.S., et al, 1999, MNRAS, 318, 381

Yudin, B., Balega, Y.Y., Blöcker, T., Hofmann, K.-H., Schertl, D., \& Weigelt, G., 2001, A\&A379 229 